\title{
Exotic pest research goes high-tech
}

alifornia's battle against exotic pests will go high-tech on April 11, when UC Riverside opens a state-of-the-art facility permitting scientists to expand use of exotic organisms in biological control research, and to carry out related genetic engineering.

The new Insectary and Quarantine Facility will nearly triple UC Riverside's capacity to serve the Western states and the nation in the importation, evaluation and rearing of natural enemies. The physical containment facilities will enable scientists to safely investigate exotic parasites, microorganisms and predators, as well as genetically engineered organisms such as more potent microbial insecticides.

Research on natural enemies and exotic pests, if performed in a highly secured facility, could yield vital information about exotic pests that pose threats to the state and nation. To date, the lack of up-to-date quarantine facilities has restricted the importation of exotic beneficial insects for research; the lack of secure containment facilities has virtually prohibited the use of exotic pests for research.

"When a new pest is introduced to one area of the state, we can investigate the exotic pest in a contained laboratory, learning the extent of its potential damage, and how best to control it," says Tim Paine, chair of UC Riverside's entomology department.

"It may also be possible to conduct research that will help prevent exotic pests from becoming established in California. For example, if we can study the behavior and biology of an exotic insect such as Asian longhorned beetle, we may develop better trapping technologies or better approaches to early detection, interception or eradication."

Since 1955, a new insect pest has been introduced into California every 60 days. Exotic pests cost California an estimated $\$ 3$ billion each year, or more than $\$ 100$ per capita annually. Nationally, pests and diseases account for a $25 \%$ annual loss to agricultural production.

UC Riverside's \$15 million facility will replace the campus' existing insectary, originally built in 1930 and expanded in 1960. Recognition that scientists at UC, and throughout the western United States, were operating with antiquated facilities inspired a 1991 initiative spearheaded by the UC Division of Agriculture and Natural Resources (DANR). DANR secured funding from the U.S. Department of
Agriculture and the California Department of Food and Agriculture (CDFA). UC Riverside's College of Natural and Agricultural Sciences provided the balance of construction costs.

The Riverside facility will soon be complemented by a sister facility at UC Davis, which is scheduled to break ground in April and will cost about the same. They will have similar equipment, allowing arthropod research, plant disease research and plant genome modification. The two facilities may emphasize different research areas.

The UC Riverside facility will consist of two receiving rooms, six research laboratories, 12 greenhouses, three environmental rooms and 64 rearing rooms. Each of these rooms must have its own climate control - meaning temperature, humidity and lighting that can closely mimic climatic conditions hospitable to insects brought here from all over the world.

"The facility is ingeniously designed to prevent exotic or genetically modified organisms from escaping to the outside environment, and to prevent contamination between laboratories," Paine says. "For instance, the building consists of three floors and three interstitital spaces between and above floors, allowing building maintenance and repairs to be done without workmen ever entering quarantine spaces."

Secured areas are sealed by magnetically controlled, air-locked double doors. Air pressure is greater in the hallway than in rooms so that when doors open, insects or other organisms will not be carried out on air currents. All air into and out of the laboratories and greenhouses will be filtered, screening microorganisms and pollen. Equipment and solid waste leaving the facility will be decontaminated.

Before bringing exotic organisms such as insects into the facility, researchers must receive permits from the USDA and CDFA, as well as approval from an internal review committee. Once granted, researchers must adhere to a strict protocol for movement of insect and plant materials. - Jaghtwhite

\begin{abstract}
Workers put the finishing touches on the new \$15 million Insectary and Quarantine Facility at UC Riverside, top. It replaces the 1930 entomology building, below, which has been deemed seismically unsafe.
\end{abstract}
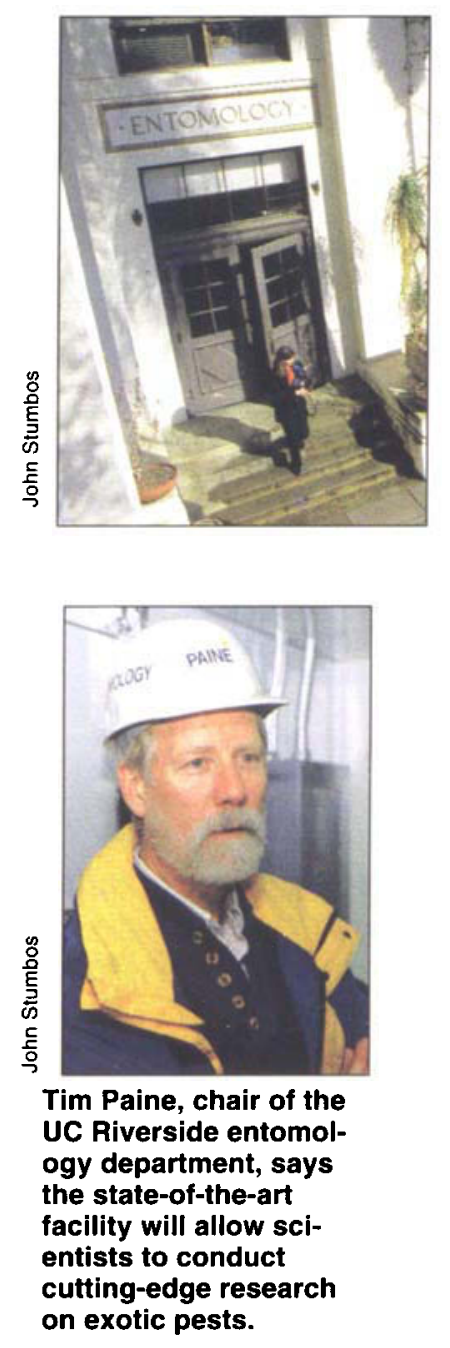